\title{
Commentary Who is at increased risk for acute kidney injury following noncardiac surgery?
}

Patrick Murray

Professor, UCD School of Medicine and Medical Science, Consultant in Nephrology and Clinical Pharmacology, Mater Misericordiae University Hospital, Nelson Street, Dublin 7, Ireland

Corresponding author: Patrick Murray, patrick.murray@ucd.ie

Published: 30 July 2009

Critical Care 2009, 13:171 (doi:10.1186/cc7942)

This article is online at http://ccforum.com/content/13/4/171

(c) 2009 BioMed Central Ltd

See related research by Abelha et al., http://ccforum.com/content/13/3/R79

\begin{abstract}
Abelha and colleagues evaluated the incidence and determinants of postoperative acute kidney injury (AKI) after major noncardiac surgery in patients with previously normal renal function. In this retrospective study of 1,166 patients with no previous renal insufficiency, who were admitted to a postsurgical intensive care unit (ICU) over a 2-year period, the incidence of AKI was $7.5 \%$. Multivariate analysis identified American Society of Anesthesiologists physical status, Revised Cardiac Risk Index, high-risk surgery and congestive heart disease as preoperative AKI risk factors. AKI was an independent risk factor for hospital mortality (odds ratio $=3.12,95 \%$ confidence interval $=1.41$ to 6.93 ; $P=0.005)$, and was associated with higher severity of illness scores (Simplified Acute Physiology Score II and Acute Physiology and Chronic Health Evaluation II), longer ICU length of stay, higher ICU mortality, increased hospital mortality and higher mortality at 6 month follow up. Although the study design excluded 121 patients with significant preoperative renal insufficiency by design, the relatively crude serum creatinine cut-offs used certainly permitted inclusion of numerous patients with preoperative renal impairment. Accordingly, the study design failed to quantify the impact of preoperative renal impairment on risk and outcomes of perioperative $\mathrm{AKI}$ in noncardiac surgery, and this should be a goal of such studies in the future. Nonetheless, the study is an important addition to the literature in an under-studied population of patients at high risk for AKI.
\end{abstract}

Acute kidney injury (AKI) has long been recognized as a devastating surgical complication [1,2]. Despite the widespread recognition of an increased risk for AKI following a variety of surgical procedures (coronary artery bypass, cardiac valve replacement, aortic aneurysm repair, and other major and/or emergent procedures), the pathogenesis of this syndrome is poorly understood in all of these settings. Accordingly, no interventions have conclusively been proven to prevent perioperative $\mathrm{AKI}$, or to ameliorate the course and outcome of evolving $\mathrm{AKI}$ identified during the early postoperative period [3].

Efforts to identify approaches to decrease the incidence of perioperative $\mathrm{AKI}$ rely upon careful characterization of risk factors, in order to appropriately target clinical management and prophylactic therapies in clinical trials [4]. Studies of perioperative cohorts can identify factors associated with the development and severity of AKI, and a subset of these are modifiable. Modifiable risk factors are further subdivided into preoperative, operative and postoperative categories. It is critically important that multicentre cohorts of patients at high risk for perioperative $\mathrm{AKI}$ are studied to inform the design of successful clinical trials of approaches to prevent and treat AKI in surgical patients. Most such studies have been conducted in cardiac surgery cohorts, many of them in single centres [5] and some in larger multicentre registries [6].

In the previous issue of Critical Care, Abelha and colleagues [1] presented the results of an analysis of AKI incidence and risk factors in a single-centre study of 1,166 adults undergoing noncardiac surgery and admitted to a postsurgical intensive care unit (postanaesthesia care unit [PACU]) over a 2-year period. They excluded patients who did not have a serum creatinine determination within 30 days before surgery. They further excluded those with evidence of preoperative renal dysfunction (defined as a requirement for renal replacement therapy or a preoperative serum creatinine $>1.6 \mathrm{mg} / \mathrm{dl}$ for men and $>1.4 \mathrm{mg} / \mathrm{dl}$ for women, within 30 days preoperatively). They retrospectively assessed which factors were predictive of $\mathrm{AKI}$, defined by stage 1 of the Acute Kidney Injury Network classification (increment of serum creatinine $\geq 0.3 \mathrm{mg} / \mathrm{dl}$ or $\geq 50 \%$ from baseline within

$\mathrm{AKI}=$ acute kidney injury; CKD = chronic kidney disease; GFR = glomerular filtration rate; PACU = postanaesthesia care unit; RCRI = Revised Cardiac Risk Index. 
48 hours or urine output $<0.5 \mathrm{ml} / \mathrm{kg}$ per hour for $>6$ hours despite fluid resuscitation).

They found that $87(7.5 \%)$ developed AKI. Univariate analysis identified age, American Society of Anesthesiologists physical status, emergency surgery, high-risk surgery (defined as intraperitoneal, intrathoracic, or suprainguinal vascular procedures), ischaemic heart disease, congestive heart disease and the Revised Cardiac Risk Index (RCRI) score as preoperative predictors of $\mathrm{AKI}$ in the postoperative period. The RCRI score includes the following variables: high-risk surgery (as defined above), ischaemic heart disease, congestive heart failure, cerebrovascular disease and insulinrequiring diabetes mellitus). Multivariate analysis eliminated age, emergency surgery and ischaemic heart disease, leaving American Society of Anesthesiologists physical status, RCRI score, high-risk surgery and congestive heart failure as the independent preoperative risk factors for AKI during the postoperative period.

Patients who developed AKI had higher Simplified Acute Physiology Score II and Acute Physiology and Chronic Health Evaluation II score, and worse outcomes: longer stay in the PACU, higher PACU mortality, higher hospital mortality and higher mortality at 6-month follow up. Finally, AKI was an independent risk factor for hospital mortality (odds ratio $=$ $3.12,95 \%$ confidence interval $=1.41$ to $6.93 ; P=0.005$ ).

The AKI risk factors identified in this study are similar to those found in other recent cohorts of patients undergoing noncardiac surgery, such as those discussed in the report by Abelha and colleagues $[7,8]$. However, the study has several limitations, most of which are acknowledged by the authors.

The most important limitation is the exclusion of patients with preoperative renal dysfunction, which has been identified as a major risk factor for perioperative AKI in most studies $[5,6]$. Indeed, chronic kidney disease (CKD) has more broadly been identified as a significant risk factor for the development of $\mathrm{AKI}$, and $\mathrm{AKI}$ has been identified as an important accelerator of CKD progression [9]. It would have been interesting to determine the contribution of prior CKD to the increased perioperative risk for AKI in this study cohort, but 121 such patients were excluded per protocol. On the other hand, the relatively crude criteria used to exclude patients with preoperative CKD (serum creatinine cut-offs adjusted for sex but not age or race) certainly failed to exclude many patients with significantly decreased glomerular filtration rate (GFR) and normal or mildly elevated serum creatinine values. Accordingly, the study did not examine the contribution of pre-existing CKD to the risk and outcomes of perioperative AKI, whether occurring in those with severe CKD (excluded from the study) or in those with lesser levels of CKD (included in the study by liberal protocol cut-offs, but not subjected to any analysis as a discrete subgroup). Other study limitations are also acknowledged, such as the lack of detailed records to determine the potential contribution of operative and postoperative factors, such as the use of nephrotoxins (nonsteroidal anti-inflammatory drugs, some forms of hydroxyethyl starch and so on) to AKI risk.

Nonetheless, the study makes an important contribution to the effort to define risk factors for AKI after noncardiac surgery. It is important that such efforts are expanded to multicentre registries, and that careful stratification of preoperative renal function is included in these studies. Specifically, it has been suggested that preoperative baseline serum creatinine values are used to determine an estimated GFR based on the MDRD (modification of diet in renal disease) equation or other estimated GFR calculators in each patient [4]. Also, perioperative records should include careful recording of exposure to nephrotoxic insults. Finally, it is suggested that the impact of perioperative AKI on long-term renal function should also be examined in large prospective databases, because the development of new CKD ( 3 months of sustained GFR $<60 \mathrm{ml} /$ minute per $1.73 \mathrm{~m}^{2}$ body surface area or evidence of kidney damage, such as proteinuria) or accelerated GFR loss superimposed on prior CKD are other clinically significant sequelae of AKI [4,9]. Hopefully, the identification of a robust score to predict $\mathrm{AKI}$ after major surgery will lead to successful clinical trials of prophylactic strategies to prevent this devastating clinical syndrome.

\section{Competing interests}

The author declares that they have no competing interests.

\section{References}

1. Abelha FJ, Botelho M, Fernandes V, Barros H: Determinants of postoperative acute kidney injury. Crit Care 2009, 13:R79.

2. Jones DR, Lee HT: Perioperative renal protection. Best Pract Res Clin Anaesthesiol 2008, 22:193-208.

3. Tang IY, Murray PT: Prevention of perioperative acute renal failure: what works? Best Pract Res Clin Anaesthesiol 2004, 18:91-111.

4. Murray P T, Devarajan P, Levey AS, Eckardt KU, Bonventre JV, Lombardi R, Herget-Rosenthal S, Levin A: A framework and key research questions in AKI diagnosis and staging in different environments. Clin J Am Soc Nephrol 2008, 3:864-868.

5. Thakar CV, Arrigain S, Worley S, Yared JP, Paganini EP: A clinical score to predict acute renal failure after cardiac surgery. $J$ Am Soc Nephrol 2005, 16:12-14.

6. Mangano CM, Diamondstone LS, Ramsay JG, Aggarwal A, Herskowitz A, Mangano DT: Renal dysfunction after myocardial revascularization: risk factors, adverse outcomes, and hospital resource utilization. Ann Intern Med 1998, 128:194-203.

7. Kheterpal S, Tremper KK, Englesbe MJ, O'Reilly M, Shanks AM, Fetterman DM, Rosenberg AL, Swartz RD: Predictors of postoperative renal failure after noncardiac surgery in patients with previously normal renal function. Anesthesiology 2007, 107:892-902.

8. Kheterpal S, Tremper KK, Heung M, Rosenberg AL, Englesbe M, Shanks AM, Campbell DA Jr: Development and validation of an acute kidney injury risk index for patients undergoing general surgery: results from a national data set. Anesthesiology 2009, 110:505-515.

9. Okusa MD, Chertow GM, Portilla D for the Acute Kidney Injury Advisory Group: The nexus of acute kidney injury, chronic kidney disease, and world kidney day 2009. Clin J Am Soc Nephrol 2009, 4:520-522. 